\title{
Method of an Operative Estimation \\ of Radio-Electronic Conditions in Interests \\ of Maintenance of Reserve \\ and Electromagnetic Compatibility \\ of Radio-Electronic Means
}

\author{
Yuri L. Koziratsky, \\ Alexey V. Ivantsov* and Ervand A. Mamadganyan \\ Military Education and Research Centre of Military-Air Forces \\ «Military-Air Academy \\ Named After Professor N.E. Zhukovsky and Yu.A. Gagarin» \\ 54a Starykh Bol'shevikov Str., Voronezh, 394064, Russia
}

Received 15.03.2016, received in revised form 29.10.2017, accepted 04.01.2018

The method of an operative estimation of radio-electronic conditions in interests of maintenance of reserve and electromagnetic compatibility of radio-electronic means in grouping is developed. The method is based on representation in a matrix kind of components of level of unmasking radiation (inadvertent) hindrances from each radio-electronic means that has given the chance to consider individually each of operating factors and to estimate its influence.

Keywords: an estimation of radio-electronic conditions, electromagnetic compatibility.

Citation: Koziratsky Yu.L., Ivantsov A.V., Mamadganyan E.A. Method of an operative estimation of radio-electronic conditions in interests of maintenance of reserve and electromagnetic compatibility of radio-electronic means, J. Sib. Fed. Univ. Eng. technol., 2018, 11(3), 256-262. DOI: 10.17516/1999-494X-0037.

(C) Siberian Federal University. All rights reserved

* Corresponding author E-mail address: kagan13@yandex.ru 


\title{
Метод оперативной оценки радиоэлектронной обстановки \\ в интересах обеспечения скрытности \\ и электромагнитной совместимости \\ радиоэлектронных средств
}

\author{
Ю.Л. Козирацкий, А.В. Иванцов, Е.А. Мамаджанян \\ Военный учебно-научный центр Военно-воздушных сил \\ «Военно-воздушная академия \\ имени профессора Н.Е. Жуковского и Ю.А. Гагарина» \\ Россия, 394064, Воронеж, ул. Старых Большевиков, 54 а
}

\begin{abstract}
Разработан метод оперативной оценки радиоэлектронной обстановки в интересах обеспечения скрытности и электромагнитной совместимости радиоэлектронных средств в группировке. Метод основан на представлении в матричном виде составляющих уровня демаскирующего излучения (непреднамеренных) помех от каждого радиоэлектронного средства, что дало возможность индивидуально учитывать каждый из действующих факторов и оценивать его влияние.
\end{abstract}

Ключевые слова: оценка радиоэлектронной обстановки, электромагнитная совместимость.

Современный уровень развития связи и телекоммуникаций характеризуется большой насыщенностью территории радиоэлектронными средствами (РЭС) различного назначения, имеющими высокую плотность размещения, даже одновременного размещения нескольких РЭС в одном месте [1]. Наиболее ярко это проявляется во время проведения различных массовых мероприятий (например, Олимпиады-2014), действиях МЧС при ликвидации стихийных бедствий. Вместе с тем развитие элементной базы ведет к увеличению чувствительности РЭС, а суммарная ширина используемого радиочастотного спектра (РЧС) остается неизменной. Существующие же методы борьбы с внеполосными и побочными излучениями как по основным, так и по боковым лепесткам диаграмм направленности антенны (ДНА) достигли максимума своих возможностей. Особенную сложность представляет обеспечение ЭМС группировок РЭС при воздействии естественных и искусственных помех различной природы.

К настоящему времени все РЭС локации, связи и навигации имеют возможность весьма точного определения своих координат, состояния (режима работы), характеристик и других необходимых сведений. С использованием технологий совместной обработки информации эти данные могут поступать на некоторый пункт управления, построенный на использовании достижений геоинформационных технологий и обеспечивающий на основе получаемой информации, собственного информационного обеспечения и заложенных в него алгоритмов выработку количественной информации по обеспечению электромагнитной совместимости этой совокупности РЭС и снижению общего уровня электромагнитного излучения при заданном уровне устойчивой работы группировки РЭС. Понятно, что основу такого управления должны составлять алгоритмы оценки радиоэлектронной обстановки и поиска РЭС-источников непреднамеренных помех (РЭС-ИНП) [2]. Однако существующие методики оценки ЭМС [2-4] носят преимущественно инженерную или исследовательскую направленность и слабо ориентирова-

$$
-257-
$$


ны на оперативное использование в условиях сложной быстроменяющейся радиоэлектронной обстановки.

Целью настоящей статьи является разработка метода оперативной оценки РЭО в интересах обеспечения скрытности и ЭМС группировки РЭС, позволяющего на основе определения уровня демаскирующего излучения (непреднамеренных помех) от каждой РЭС оперативно управлять организационными и техническими параметрами их функционирования.

Примем, что на определенной территории функционирует $m$ РЭС, каждое из которых имеет приемное и передающее устройства, размещенные в одной точке. Все средства характеризуются априори известными параметрами размещения, включающими географические координаты $x_{i}, y_{i}$ и высоту над уровнем моря, с учетом высоты подъема антенны $z_{i}$.

Пример взаимного размещения РЭС при $m=6$ представлен на рис. 1.

Каждое $i$-е РЭС характеризуется следующими априори известными параметрами:

1) техническими параметрами РЭС:

выходной мощностью РЭС $P_{\text {выхі; }}$

амплитудной диаграммой направленности передающей антенны $F_{1}\left(\theta_{i j}\right)$;

амплитудной диаграммой направленности приемной антенны $F_{2}\left(\theta_{j i}\right)$;

коэффициентом усиления передающей антенны $G_{1 i}$;

коэффициентом усиления приемной антенны $G_{2 i}$;

несущей (центральной) частотой $f_{i}$;

шириной спектра сигнала $\Delta f_{c i}=f_{\mathrm{в} i}-f_{\text {нi; }}$;

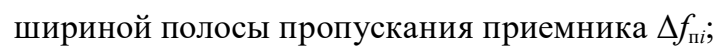

Каждая $i j$-я пара РЭС характеризуется следующими параметрами совместного функционирования:

расстоянием между $i$-м и $j$-м РЭС $R_{i j}\left(R_{i j}^{2}=\left(x_{i}-x_{j}\right)^{2}+\left(y_{i}-y_{j}\right)^{2}+\left(z_{i}-z_{j}\right)^{2}\right)$;

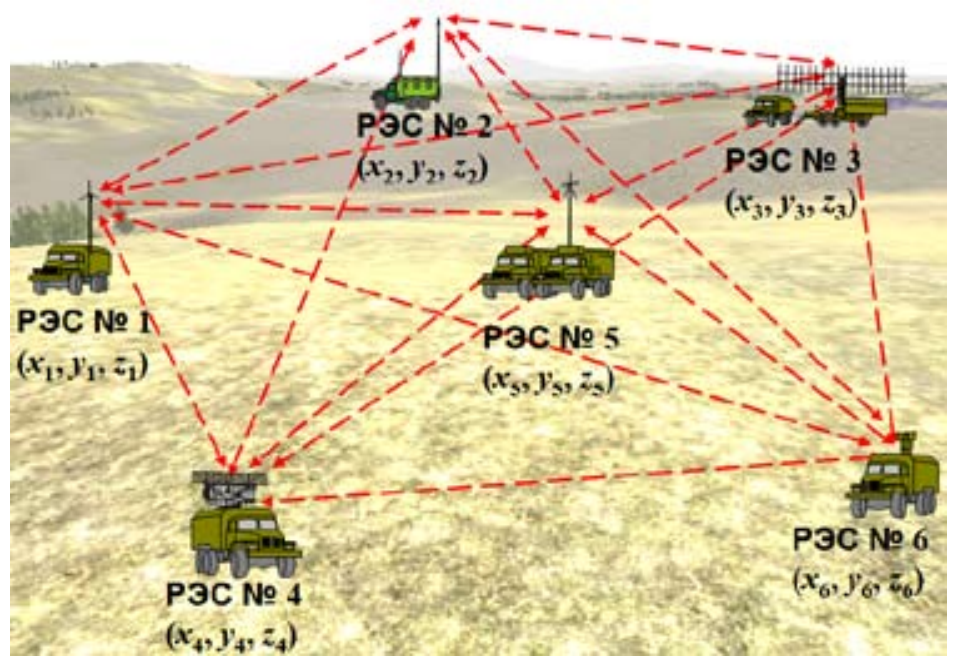

Рис. 1. Пример взаимного расположения РЭС в группировке

Fig. 1. Example of radio-electronic equipment mutual disposition in a group 
коэффициентом рассогласования по поляризации $k_{i j}(\gamma)$;

коэффициентом рассогласования по частоте $K_{i j}(f)$;

коэффициентом затухания на трассе между $i$-м и $j$-м РЭС $T\left(R_{i j}\right)$.

Коэффициенты затухания на трассе $T\left(R_{i j}\right)$ определяются по известным методикам $[3,4]$ исходя из характеристик РЭС и профиля трасс распространения между $i$-м и $j$-м РЭС. Значение коэффициента рассогласования по поляризации $k_{i j}(\gamma)$ (от 0 до 1 ) определяется углом $\gamma$ между направлениями поляризации непреднамеренной помехи и антенны (РЭС-ОВП).

Коэффициент рассогласования по частоте $K_{i j}(f)$ можно вычислить так:

$$
K_{i j}(f)=\frac{\int_{-\infty}^{\infty} H^{2}(f) S^{p}(f) d f}{\int_{-\infty}^{\infty} S^{p}(f) d f} .
$$

Мощность сигнала $i$-й РЭС как источника непреднамеренных помех (РЭС-ИНП) на входе приемника $j$-го РЭС-объекта воздействия помех (РЭС-ОВП) на основе приведенных выше исходных данных определяется выражением [5]

$$
P_{i j}=P_{\text {вых } i}\left(\frac{\lambda}{4 \pi \cdot R_{i j}}\right)^{2} F_{1}^{2}\left(\theta_{i j}\right) \cdot F_{2}^{2}\left(\theta_{j i}\right) \cdot G_{1 i} \cdot G_{2 i} \cdot T\left(R_{i j}\right) \cdot K_{i j}(f) \cdot k_{i j}(\gamma) .
$$

В случае попарной оценки рассматривается поочередное воздействие каждого РЭС-ИНП на каждое РЭС-ОВП соответственно. На основе (2) можно построить матрицу взаимного влияния следующего вида:

$$
\left\|P_{\mathrm{B}}\right\|=\left\|\begin{array}{ccccc}
P_{11} & P_{12} & P_{1 i} & \ldots & P_{1 m} \\
P_{21} & P_{22} & P_{2 i} & \ldots & P_{2 m} \\
P_{j 1} & P_{j 2} & P_{i j} & \ldots & P_{i m} \\
\ldots & \ldots & \ldots & \ldots & \ldots \\
P_{m 1} & P_{m 2} & P_{m j} & \ldots & P_{m m}
\end{array}\right\|,
$$

где $P_{i j}$ - мощность сигнала $i$-й РЭС на входе приемника $j$-го РЭС, определяемая выражением (2).

Очевидно, что составляющие главной диагонали матрицы должны показывать воздействие РЭС самой на себя и не будут учитываться в дальнейших расчетах. Кроме того, если $i$-я и $j$-я РЭС являются корреспондентами одной радиосети, то сигналы $i$-й РЭС на входе приемника j-й РЭС и, наоборот, будут не помеховыми, а полезными, и в матрице также учитываться не должны.

Таким образом, матрица (3) преобразуется к виду

$$
\left\|P_{\mathrm{B}}\right\|=\left\|\begin{array}{ccccc}
0 & P_{12} & P_{1 i} & \ldots & P_{1 m} \\
P_{21} & 0 & P_{2 i} & \ldots & P_{2 m} \\
P_{j 1} & P_{j 2} & 0 & \ldots & P_{i m} \\
\ldots & \ldots & \ldots & 0 & \ldots \\
P_{m 1} & P_{m 2} & P_{m j} & \ldots & 0
\end{array}\right\| .
$$


Однако полученная матрица взаимного влияния (4) требует определенной декомпозиции для доступного представления всех параметров и характеристик, влияющих на обеспечение ЭМС группировки РЭС.

Введем следующие показатели:

$K_{i j}$ - коэффициент частотно-поляризационного рассогласования, определяемый выражением

$$
K_{i j}=\ln K_{i j}(f) \cdot K_{i j}(\gamma)=\ln \left(K_{i j}(f) \cdot \cos \gamma\right) ;
$$

$M_{i j}$ - показатель ослабления, определяемый выражением

$$
M_{i j}=\ln \left(\left(\frac{\lambda}{4 \pi \cdot R_{i j}}\right)^{2} T\left(R_{i j}\right)\right) ;
$$

$Q_{i j}$ - коэффициент антенн, определяемый выражением

$$
Q_{i j}=\ln \left(F_{1}^{2}\left(\theta_{i j}\right) \cdot F_{2}^{2}\left(\theta_{j i}\right) \cdot G_{1 i} \cdot G_{2 i}\right) .
$$

С использованием введенных показателей и с учетом свойств логарифмов выражение (2) можно преобразовать как:

$$
\begin{aligned}
& \ln P_{i j}=\ln \left(P_{\text {вых } i}\left(\frac{\lambda}{4 \pi \cdot R_{i j}}\right)^{2} F_{1}^{2}\left(\theta_{i j}\right) \cdot F_{2}^{2}\left(\theta_{j i}\right) \cdot G_{1 i} \cdot G_{2 i} \cdot T\left(R_{i j}\right) \cdot K_{i j}(f) \cdot k_{i j}(\gamma)\right)= \\
& =P_{\text {вых } i}+M_{i j}+Q_{i j}+K_{i j} .
\end{aligned}
$$

С учетом принятых обозначений матрица взаимного влияния может быть представлена в виде суммы матриц

$$
\left\|P_{\mathrm{B}}\right\|_{m m}=\left(\left\|P_{\text {lвых }}\right\|_{m m}+\|M\|_{m m}+\|Q\|_{m m}+\|K\|_{m m}\right) .
$$

Матрица (9) определяет лишь парное взаимное влияние РЭС в группировке. Однако на каждое РЭС в группировке будут воздействовать непреднамеренными помехами различной интенсивности все РЭС. Для определения суммарной мощности непреднамеренных помех необходимо осуществить потенцирование элементов матрицы (9) и последующее умножение [7]:

$$
\left\|P_{\Sigma}\right\|_{1 m}=\ln \left\{\left\|P_{\mathrm{B}}\right\|^{\Pi} \times\|J\|_{1 m}\right\} .
$$

Для принятия решения о том, совместимы РЭС или нет, осуществляется определение суммарного воздействия всех $m-1$ РЭС на $j$-ю РЭС и сравнение его с допустимой пороговой величиной помехи на входе приемника $i$-й РЭС, которая определяет минимально допустимое для работы РЭС с требуемым качеством отношение сигнал/помеха на входе приемника РЭС. При выполнении условия $P_{\Sigma j}^{\prime}<P_{\text {пор } j}^{\prime}$ считают, что ЭМС выполнена, при $P_{\Sigma j}^{\prime} \geq P_{\text {пор } j}^{\prime}-$ ЭМС не выполнена.

На основе разработанного метода возможно составить алгоритм оценки радиоэлектронной обстановки группировки РЭС, представленный на рис. 2.

Таким образом, разработан метод оперативной оценки радиоэлектронной обстановки в интересах обеспечения скрытности и электромагнитной совместимости группировки РЭС, по- 


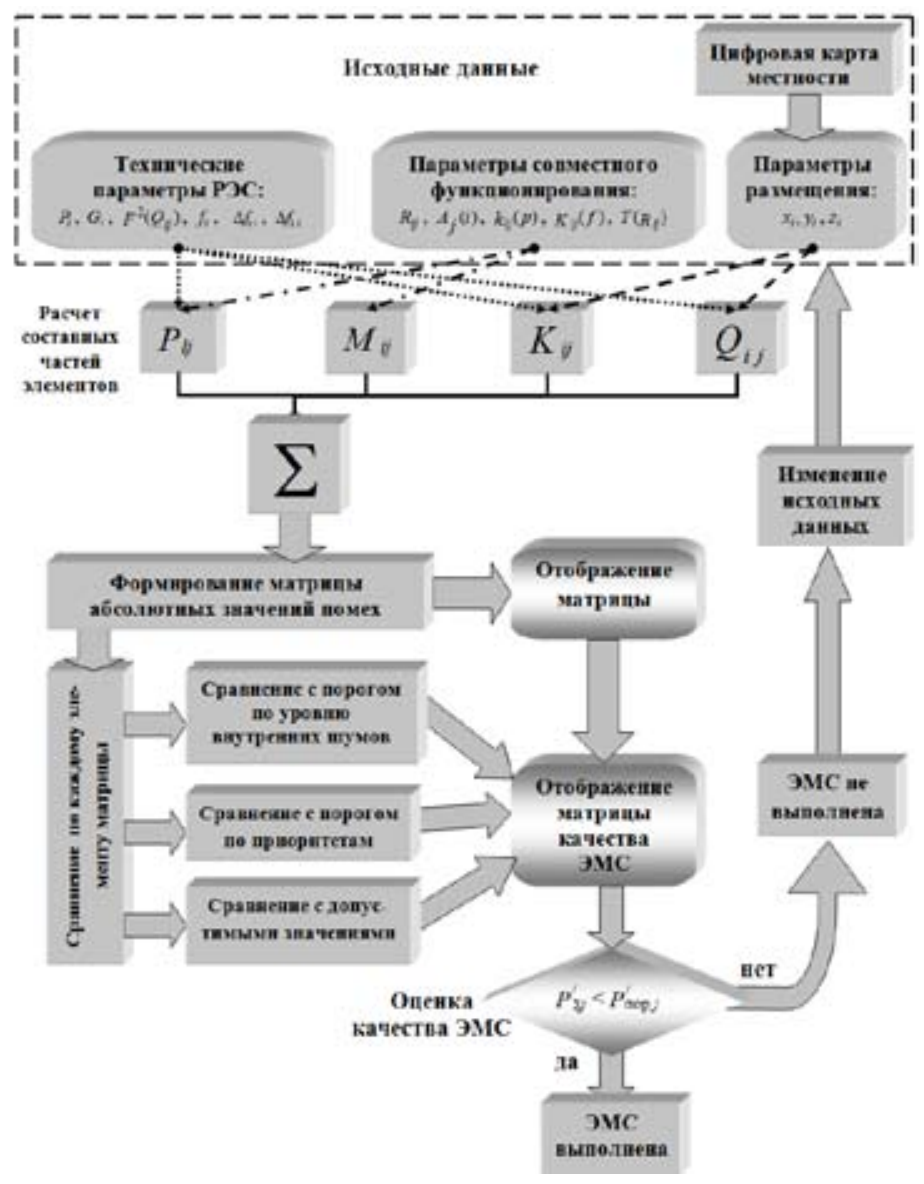

Рис. 2. Алгоритм оценки радиоэлектронной обстановки

Fig. 2. Radio-frequency environment estimation algorithm

зволяющий на основе определения суммарного уровня взаимных непреднамеренных помех РЭС оперативно управлять организационными и техническими параметрами их функционирования. Особенностью метода является представление в матричном виде составляющих уровня непреднамеренных помех от каждой РЭС, что дало возможность индивидуально учитывать каждый из действующих факторов и оценивать его влияние, а логарифмическое преобразование позволило перейти от произведения коэффициентов к их сумме.

\section{Список литературы}

[1] Модели информационного конфликта средств поиска и обнаружения; ред. Ю.Л. Козирацкий. М.: Радиотехника, 2013. 232 с. [Model information of the conflict of means of search and discovery; ed. by Ju.L. Kozirazky. M., Radiotexnika, 2013. 232 p. (in Russian)].

[2] Козирацкий Ю.Л., Сорокин А.Д., Ступницкий М.М. Управление использованием радиочастотного спектра, Вооружение, политика конверсия. 1996, 5-6, 40-45. [Kozirazky Ju.L., Sorokin A.D., Stupnitsky M.M. The spectrum management, Weapons, politics, conversion. 1996, 5-6, 40-45. (in Russian)]. 
[3] Управление радиочастотным спектром и электромагнитной совместимостью радиосистем; ред. М.А. Быховский. М.: Эко-Трендз, 2006. 376 с. [Management of radio frequency spectrum and electromagnetic compatibility of radio systems; edited by M.A. Bykhovsky. M., EKOTrendz, 2006. 376 p. (in Russian)].

[4] Липатников В.А., Кулешов И.А. Управление радиочастотным спектром. СПб.: ВАС, 2011. 384 c. [Lipatnikov V.A., Kuleshov I.A. Radio frequency spectrum control. SPb., YOU, 2011. 384 p. (in Russian)].

[5] Посохин Н.И., Сонников В.Г., Максимов Ю.Н. Радиоэлектронная борьба. СПб.: ВКА им. Можайского, 2002. 375 с. [Posokhin N.I., Sonnikov V.G., Maksimov Yu.N. Electronic warfare. SPb., VKA im. Mozhaisky, 2002. 375 p. (in Russian)].

[6] Радиолокаиионные антенные устройства. Справочник по радиолокации. Пер. с англ. Т. 2; ред. М. Сколник. М.: Сов. радио, 1977. 397 с. [Radar antenna devices. Guide to radar. Per. from English. Vol. 2; edited by M. Skolnick. M., Sov. radio, 1977. 397 p. (in Russian)].

[7] Покорная О.Ю., Ковалева М.И. Математика, аналитическая геометрия и линейная алгебра. Ч. 1. Матрицы и определители. Воронеж: ВАИУ, 2011. 41 с. [Pokornaya O.Yu., Kovaleva M.I. Mathematics, analytical geometry and linear algebra. Part 1. Matrices and determinants. Voronezh, VAIU, 2011. 41 p. (in Russian)]. 